\title{
3-D Visual Design of Casting Crane Trolley Based on Large Data
}

\author{
Yixiao Qin ${ }^{1}$, Linnan Liu ${ }^{1}$, Wei Guo ${ }^{1,2}$ \\ ${ }^{1}$ College of Mechanical Engineering, Taiyuan University of Science and Technology, Taiyuan, China \\ ${ }^{2}$ College of Mechanical Engineering, Xi'an Jiaotong University, Xi'an, China
}

Email address:

649783251@qq.com (Linnan Liu), qyx819@163.com (Yixiao Qin)

\section{To cite this article:}

Yixiao Qin, Linnan Liu, Wei Guo. 3-D Visual Design of Casting Crane Trolley Based on Large Data. Science Research. Vol. 4, No. 4, 2016, pp. 98-102. doi: 10.11648/j.sr.20160404.11

Received: July 12, 2016; Accepted: July 25, 2016; Published: August 10, 2016

\begin{abstract}
Casting cranes are special equipment to lifting the ladle in metal smelting workshop, the design of them are directly related to the safety of production. In the traditional design method, the design of the mechanical components and the choice of the calculation are time-consuming and laborious, and the design efficiency is low. Today, the parts of casting crane have standardized, and they are helpful to automatically design by using computers. In this study, a parametric design system of casting crane trolley is established by using secondary development of SolidWorks based on the large data of the basic parts. The system can realize precise design of casting crane trolley whose institution is complex. And this is helpful for the development of new models and quality improvement of this special equipment.
\end{abstract}

Keywords: Casting Crane Trolley, Secondary Development of SolidWorks, Large Data of the Basic Parts, Parametric Design

\section{Introduction}

As an important equipment for transporting molten metal, the casting crane plays an important role in the process of smelting. With the improvement of the automation of large metallurgical enterprises, the safety and stability of the casting cranes are more strict. The development trend of the modern foundry crane is the modularization of structure, automation of design, multi speed frequency conversion, intelligent operation and convenient maintenance. Konecrane in Finland, Eurocrane in Germany, Stahl in Germany and Scania in Sweden and other European crane manufacturing companies started early. After developmenting for a long time, they formed a complete industry system. The Able, Westinghouse and CM group in United States are also in the leading position in the development and technical innovation of casting cranes. Casting cranes made in Europe and United States have innovated in the application of the whole lightweight, modular structures and safety of cranes. And all of these make casting cranes more and more reliable. Efficient design and manufacturing of G3.0 have been realized. Intelligent design and manufacturing of G4.0 will be realized sooner or later. Then the material will be saved in the manufacturing process and energy will be saved in the running process [1]. In recent decades, Taiyuan Heavy Machinery Group Co., Ltd. and Dalian Heavy Industry Group Co., Ltd. and other crane manufacturers are adopting international advanced technology and improving the ability of independent development and innovation continuously at the same time [2].

In order to meet the needs of the development of crane industry in China, this study achieves the accurate and rapid design of complex casting crane trolley. Based on large data of the parts, this study uses programming and visualization technology on computer to realize the goal. By giving the original parameters of the casting cranes which have been designed to the software interface, the design results of the mechanism can be gotten. According to the calculation data of selected parts, software compares the design parameters of each components and selects the parts of the lifting mechanism and the operation of the trolleys. Based on parameters of selected parts, software uses VB to start SolidWorks to draw the 3-D models of the parts and assembles to 3-D assembly mechanism. In the case that assembly 
drawings are reasonable and complete, the software automatically generates 2-D CAD engineering drawings and outputs specifications of design calculation. This study sends the tasks of the design calculation and mapping of the cranes which spend much manpower and time to the computers. This transformation not only speeds up the design process and saves the labor force resources, but also can eliminate the artificial error caused by various factors. So it improves the efficiency of design and makes the accuracy higher.

\section{3-D Visualization Design of Casting Crane Trolley Based on Large Data}

\subsection{3-D Design of SolidWorks}

SolidWorks is a integration software system based on the windows desktop $\mathrm{CAD} / \mathrm{CAE} / \mathrm{CAM} / \mathrm{PDM}$. Its design process which has relevance can modify the design at any stage of the design process and affect the changes of the relevant parts at the same time. SolidWorks can simulate the process of assembling. It can also check the static interference and calculate the quality of characteristics, such as the center of mass, moment of inertia, etc. It combines 2-D drawing and 3-D modeling technology together to simplify the process of generation of engineering drawing. It has a good development of interface and function expansion, and achieves transition and transmission of the data between different kinds of CAD software easily [3-4].

In order to make it easy for secondary development, SolidWorks provides hundreds of API (application program interface and application program interface) function. When using SolidWorks API, datum and datum axis in various parts of the assemblies are established to enable the assembly process quickly and accurately. Then they can be assembled by the relationship between the datum plane or datum axis [5]. After the completion of 3-D parts' drawing, the parts should be assembled to form a complete assembly. The idea of parametric assembly and parametric modeling are basically the same. Before the VB operating SolidWorks to assemble parts, the VB and SolidWorks assembly API should be connected [6-8].

In the selection of the system design, a large number of tables should be found to determine the various coefficients. The performance and size of each components can be reflected to the designers by the form of tables. The design establishes access database, and shows out 3-D models by Cortona 3-D engine. Then according to the engineering diagram templates which are given before, it generates 2-D engineering drawings and word instructions which can provide reference for the design.

\subsection{Parametric Design System of Casting Crane Trolley}

\subsubsection{System Structure and Work Flow}

The calculation method and the model drawing method of the main lifting mechanism and the auxiliary lifting mechanism of the casting crane trolley are basically the same.
So the calculation and modeling modules are made by using the class modules in VB. It can avoid duplication of codes and improve the readability and logic of the program. One of the major functions of this system is to draw the 3-D parts drawings, assembly drawings and engineering drawings automatically, based on the parameters from users. The parameterized design system of the casting crane trolley is from top to bottom. Firstly, it determines the overall structure of the crane. Then it determines the structures of parts. Finally it generates the engineering drawings and specifications. This will facilitate precision design and improve the performance of the products. The system structure is shown in figure 1 .

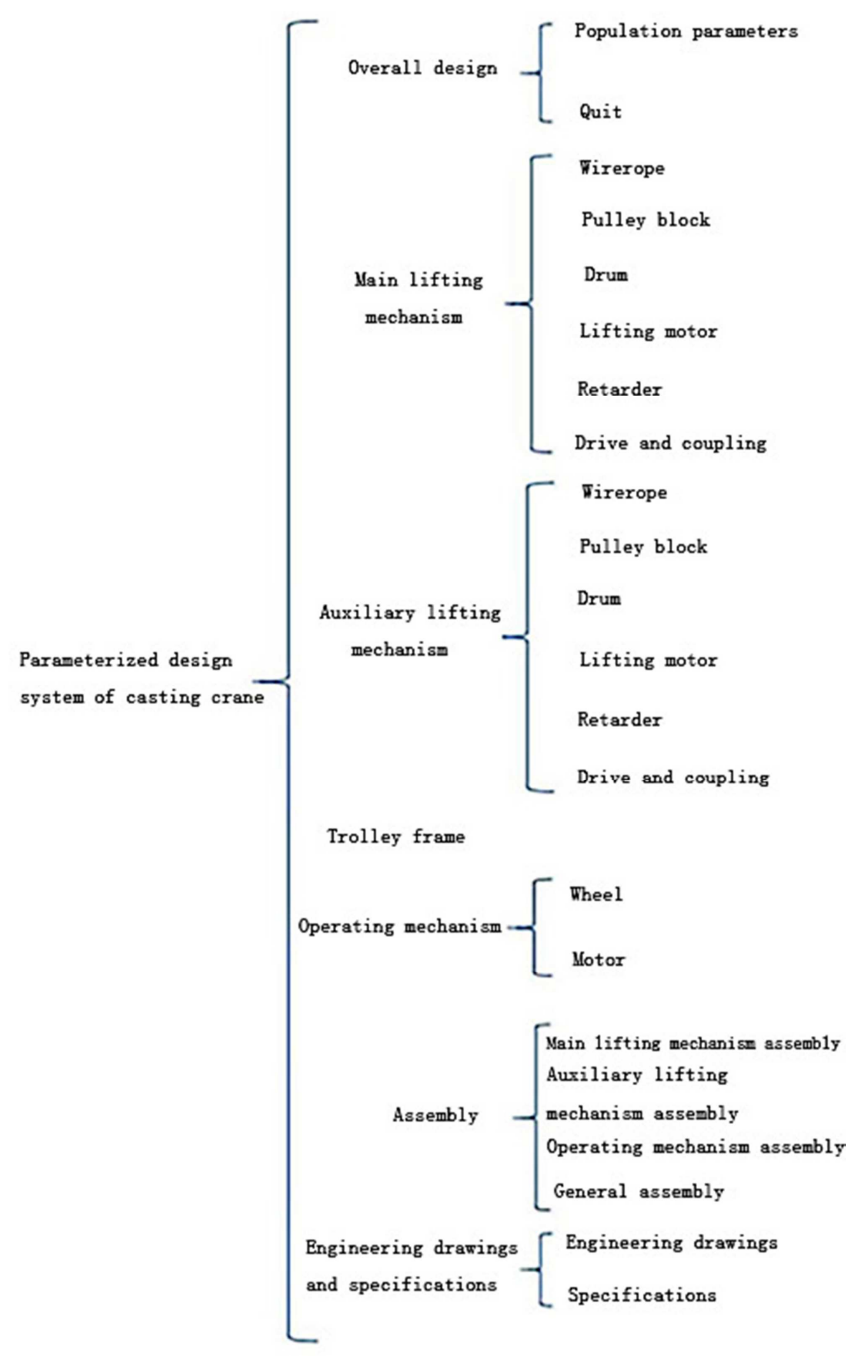

Figure 1. Configuration diagram of design system.

The workflow of this system is the main line. First of all, the system determines the structures of the casting crane trolley, mainly about the designed structures and positions of the drums, motors and brakes from the assembly. Then the system selects the appropriate components by the calculation and draws their 3-D structures. Secondly, according to the 3-D structures of the parts and the assembly between them, the system creates the assembly. Finally it outputs the project drawings and instructions. The workflow of the system is 
shown in figure 2.

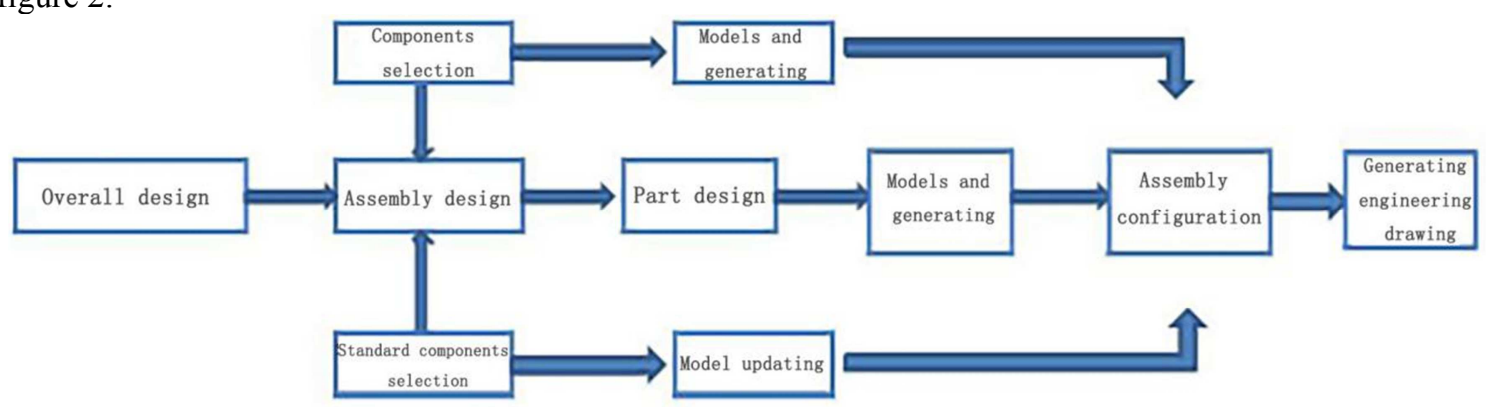

Figure 2. System workflow.

\subsubsection{Large Data Information of the Standard Parts of the Lifting Mechanism and Operation Mechanism}

The large amounts of data of standard components which support the visualization design are formatted for storage in the third standard parts database of performance table of the main and auxiliary lifting mechanism and the operating mechanism in Figure 1. It includes the performance data of wire ropes, drums, crane special motors, retarders, couplings, brakes, wheels and safety device of lifting mechanism and operating mechanism. The large amount of data can be readily updated and expanded with the product upgrading and technological progress.

\subsubsection{Parametric Design Module of Casting Crane Trolley}

The design module of the parameterized design of the casting crane trolley consists of three parts, the lifting mechanism, the operating mechanism and the travelling gear.

The lifting mechanism of the casting crane trolley can be divided into the main lifting mechanism and the auxiliary lifting mechanism. As the design method and principle of the main lifting mechanism and the auxiliary lifting mechanism are the same, the main lifting mechanism is an example to illustrate the design method. The design of the main lifting mechanism includes: the selection of wire ropes, the selection of pulley blocks, the selection of drums, the selection of retarders, the selection of motor brakes and the selection of safety devices and the couplings.

Operating mechanism of the casting crane can be divided into two types. One is running on the rail and the other is not. The operation mechanism of bridge crane, gantry crane, portal crane and tower crane are basically in the form of rail. The design of the travelling gear of the casting crane trolley mainly includes: the design of the wheels and the design of the three in one motor (motor, reducer and brake). After the selection of the operation mechanism, whether the start time of the trolley which is full or empty is within the specified time range and whether the empty trolley skids are required to be checked. Finally the number of buffers and whether buffers can take effect in cushioning stroke should be determined.

The design of safety devices of the traveling mechanism includes the design of the buffer and the calculation of operating and the non slip condition.

The functional structure of the trolley frame can be mainly divided into the following 3 parts: the beam, the platform and the base. The beam is the main supporting parts of the trolley frame. It mainly supports the main load which is passed from lifting mechanism and operation mechanism to the trolley frame. Platform is the main brace of the trolley frame. And Workers can walk on it. Base is a connecting piece of motors, reducers, brakes and the trolley frame of lifting mechanism and operating mechanism.

\subsubsection{Parametric Assembly Module of Trolley}

When the design and selection of the parts of the trolley are completed, the parts which are not linked together and ultimately should be assembled to generate a complete trolley. In the assembly process, not only the relative position between the various parts should be take into account, but also some of the interference between the parts should be avoided.

\subsubsection{Design Module of Engineering Drawings and Specifications}

When the assembly of the parts of the trolley is completed, the 3-D assembly drawings can show the relationship between the various components visually. The assembly can be printed into 2-D engineering drawings in order to check the name of the parts, the relationship between the structures and the mate-relationship easily. And it can generate a design specification to explain the structures of the engineering drawings at the same time. This can realize a set of quick design of casting crane trolleys.

\section{Application}

\begin{tabular}{|c|c|c|c|c|c|c|c|}
\hline $\begin{array}{l}\text { Overall } \\
\text { design }\end{array}$ & $\begin{array}{l}\text { Main lifting } \\
\text { mechanism }\end{array}$ & $\begin{array}{c}\text { Auxiliary lifting } \\
\text { mechanism }\end{array}$ & $\begin{array}{l}\text { Operating } \\
\text { mechanism }\end{array}$ & $\begin{array}{l}\text { Trolley } \\
\text { frame }\end{array}$ & Assembly & $\begin{array}{l}\text { Engineering drawings } \\
\text { and specifications }\end{array}$ & Help \\
\hline
\end{tabular}

Figure 3. System menu and submenu. 
Take the $180 / 50 \mathrm{~T}$ bridge casting crane produced by Taiyuan Heavy Machinery Group Co., Ltd. as an example, main/auxiliary hook load is $180 / 50 t$, main/auxiliary lifting height is $20 / 24 \mathrm{~m}$, main/auxiliary lifting speed is $8.8 / 18.8 \mathrm{~m} / \mathrm{min}$, the working level of main/auxiliary lifting mechanism is $\mathrm{M} 8 / \mathrm{M} 5$, the running speed of the trolley is $38.15 \mathrm{~m} / \mathrm{min}$, the working level of operating mechanism is $\mathrm{M} 7$, the working level of the machine is A8.

\subsection{Generation of the Trolley Assembly}

The assembly mode of casting crane trolley is applied by a branch to the whole. First, the system assembles the main lifting mechanism, the auxiliary lifting mechanism and operating mechanism. Then the main lifting mechanism, the lifting mechanism and the operating mechanism are assembled to the trolley frame. The assembly of main lifting mechanism and the assembly of auxiliary lifting mechanism are shown in Figure 4 and figure 5.

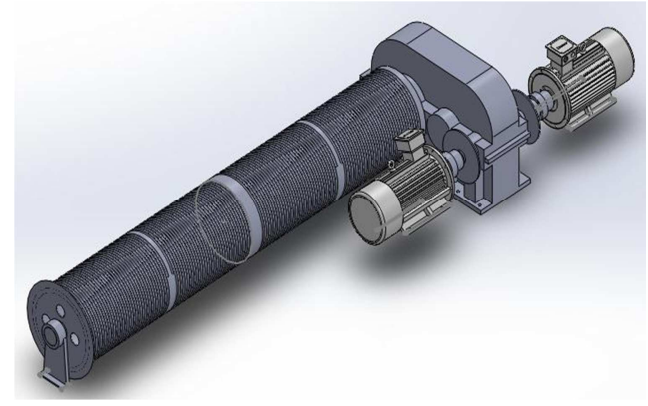

Figure 4. SolidWorks main lifting mechanism assembly.

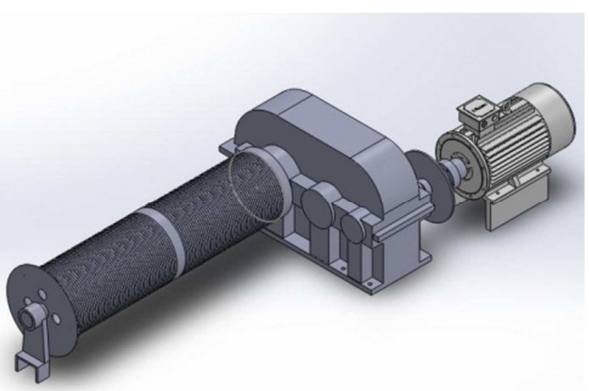

Figure 5. SolidWorks auxiliary lifting mechanism assembly.

Deceleration motors of travelling gear are installed by the foot. The base of the deceleration motors are connected with the baffle of the trolley. The weight of the casting crane trolley designed by the system is high. So there is a driver for every two wheels of the balance car. The assembly of the travelling gear is shown in Figure 6.

After finishing the design and the model drawing of all parts of the casting crane trolley, the system assembles the parts of the trolley. In the assembly process, in addition to ensure that the assembly relationship is correct, the system need to check whether the various parts produce interference or a reasonable gap. After every step of the assembly, the assembly should be checked for the interference. The trolley assembly is shown in figure 7 .

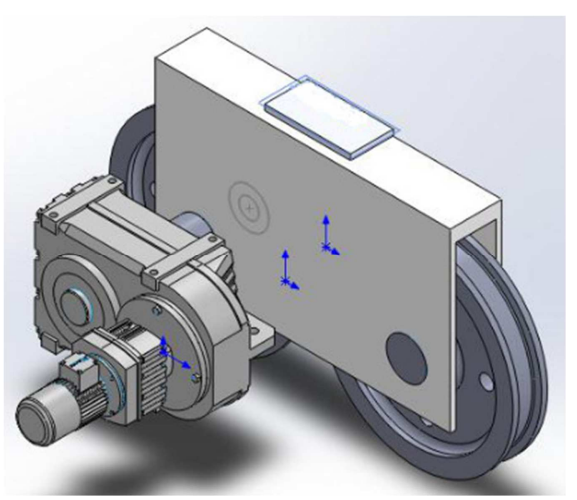

Figure 6. SolidWorks travelling gear assembly.

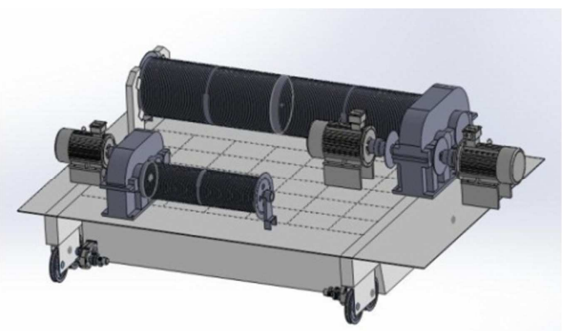

Figure 7. SolidWorks trolley assembly.

\subsection{Engineering Drawing and Specification}

After the trolley assembly is completed, in order to facilitate the use of the engineering, system generates 2-D engineering drawings. VB calls SolidWorks to generate three views automatically. Then it adds annotation and fills in the detail list and performance parameters table. Figure 8 is the 2-D engineering drawings generated by the system.

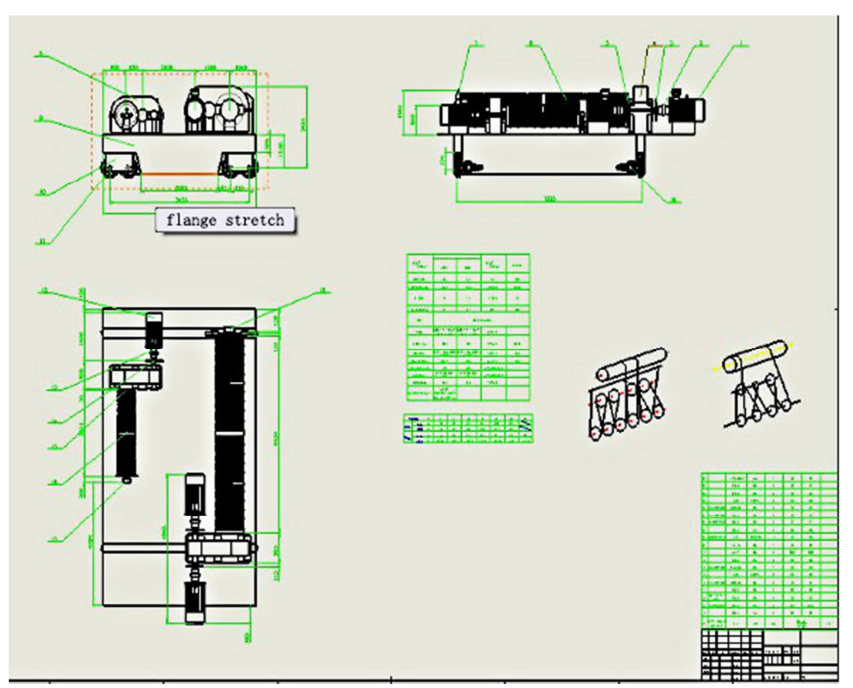

Figure 8. SolidWorks automatic generation of engineering drawings.

After click on the option of engineering drawings and instructions in the main menu, VB opens template of the design specification automatically and insert the calculated data into the corresponding position. The location where need 
us to input the data are marked by bookmarks. VB searches for bookmarks automatically and covers bookmarks with the calculated data.

\section{Conclusion}

The efficient parametric design method of the casting crane trolley has been formed in this paper. And 3-D automatic design of the casting crane trolley is realized by visual programming and access database based on large data of the basic parts. The shapes and dimensions of every selected components can be shown on the screen. By calling the API SolidWorks interface, the parts of casting crane trolley can be assembled automatically. And 2-D engineering drawings can be generated. The specification in which the calculation results are written can be output. Efficient design can be achieved by this system.

The system can realized the design of a series of casting crane trolleys. It can design or select different parts according to the different parameters of the casting cranes which are given. And it has been proven reliable and high-precision by comparing the design results with the actual casting cranes.

\section{Acknowledgments}

This work was supported by the Natural Science Foundation of Shanxi Province, China (Grant no. 2013011022-6 and no. 2014011006-2).

\section{References}

[1] Shu Bin. The structural analysis based on modern design method [J]. Proceeding 2009 IEEE 10th International
Conference on Computer-Aided Industrial Design and Conceptual Design: E-Business, Creative Design, Manufacturing - CAID and CD 2009, 2009, 22 (3): 116-118

[2] V. G. Popov, Z. K. Kabakov, D. F. Gabtykaev. Mathematical modeling of the heating of the metal structures of a foundry crane equipped with heat shields. Metallurgist, 2009. 53 (11): 704-709

[3] Andrew Burn. Making machinima: animation, games, and multimodal participation in the media arts [J]. Learning, Media and Technology, 2016, 41 (2): 310-329

[4] Yan Luximon, Roger M. Ball, Eric H. C. Chow. A design and evaluation tool using 3D head templates [J]. Computer-Aided Design and Applications, 2016, 13 (2): 153-161

[5] Anton, Daniela Tănase. Informed Geometries. Parametric Modelling and Energy Analysis in Early Stages of Design [J]. Energy Procedia, 2016, 85: 9-16

[6] Junying Ge. The Design and Realization of Visual Education System for Bridge Structure Analysis [J]. Energy Procedia, 2012, 17: 1885-1890

[7] Kazuhiko Terashima, Ying Shen, Ken'ichi Yano. Modeling and optimal control of a rotary crane using the straight transfer transformation method [J]. Control Engineering Practice, 2007, 15 (9): 1179-1192

[8] V. G. Popov, Z. K. Kabakov, D. F. Gabtykaev. Mathematical modeling of the heating of the metal structures of a foundry crane equipped with heat shields [J]. Metallurgist, 2010, 53: $11-12$

[9] Jung, Hoon-Hyung. A study on the stability of a mast structure for erecting a large crane $[\mathrm{J}]$. Applied Mechanics and Materials, 2012, 16 (3): 1483-1490

Cuiyun Gu, Xiufen Guo, Yixiao Qin. Analysis of the brake distance in the condition of ladle crane's hoisting mechanism failure [J]. Hoisting and Conveying Machinery, 2009, 12: $37-41$ 